\title{
Model for phononic energy dissipation in friction
}

\author{
A. Buldum, D. M. Leitner,* and S. Ciraci \\ Department of Physics, Bilkent University, Bilkent 06533, Ankara, Turkey
}

(Received 23 October 1998; revised manuscript received 4 December 1998)

\begin{abstract}
We have developed a microscopic model of phononic energy dissipation in friction that involves the generation of a local excess phonon distribution in a nanoparticle between two sliding objects, and its damping into the objects. The conversion of the energy stored in the nanoparticle into excess phonons and their decay rates are calculated. The model can be extended to include randomly distributed nanoparticles and phononphonon interaction through anharmonic couplings. By using this model we present a quantitative analysis of energy dissipation in sliding friction. [S0163-1829(99)03523-7]
\end{abstract}

\section{INTRODUCTION}

Recently, friction ${ }^{1}$ has become a subject of great interest owing to the atomic scale analysis provided by atomic and friction force microscopies. ${ }^{2}$ Several theoretical investigations based on simple models and atomic simulations revealed various physical phenomena, such as the stick-slip process, structural phase transitions, wear, etc. ${ }^{3-8}$ A quantitative analysis of the energy dissipation, however, has remained a major issue to be resolved. One can distinguish three different mechanisms underlying the dissipation process yet to be clarified: (i) Energy can be stored in the highformation energy phases, defects or disordered structure, which are induced through structural transitions and wear under high stress. $^{7}$ (ii) Electron-hole pairs (or perhaps charge-density waves) can be excited in sliding metal objects. ${ }^{9,10}$ It is also argued that inelastic electron scattering due to the relative motion of atoms at the interface gives rise to electronic energy dissipation. ${ }^{1,10}$ Important new data about electronic friction have been supplemented by the recent work of Dayo et al. ${ }^{11}$ who observed that the friction force due to the motion of the nitrogen layer absorbed on the $\mathrm{Pb}$ film decreases abruptly when the film is cooled below $T_{c}$. (iii) The nonequilibrium phonons that are created during sliding can be the major mechanism of energy dissipation. $^{12,13}$

In this paper we develop a model that provides a quantitative analysis of energy dissipation in friction with or without lubricants. The model is based on a microscopic theory and deals with the generation of excess (nonequilibrium) phonons and their dissipation. We first start by describing the friction system, i.e., two samples in relative motion and a nanoparticle between them, that is attached to one of the samples with a weak coupling. This system can be applied to examine the energy damping and friction force due to either the sliding of a tip (attached to a soft cantilever) over the sample in the operation of a friction force microscope (FFM) or the motion of a flake, or a small molecule in boundary lubrication. We then present our microscopic theory to analyze the generation of an excess phonon distribution in the nanoparticle as a result of the elastic deformation induced by the corrugation of sample surfaces in relative motion and its dissipation therefrom. The application of this microscopic theory on a friction system is illustrated by carrying out nu- merical calculations. We finally discuss our results together with earlier theoretical work.

\section{MODEL AND FORMALISM}

The friction system that is the subject of the present study consists of a nanoparticle including $\mathrm{N}$ atoms (an asperity or a lubricant molecule) connected to the surface of one sample (object 1), but slides over the surface of the sample (object 2) under a constant loading force $F_{N}$. This system is schematically described in Fig. 1(a). As the nanoparticle is translated from one low-energy $(H)$ site to the next one through the high-energy $(T)$ site, its potential energy increases by $\delta V_{T}$ in the interval from $H$ to $M$. This stage is called the stick step, in which the nanoparticle is elastically deformed; namely its atoms are displaced by $\left\{\mathbf{u}_{\mathrm{j}}\right\}$ whereby part of the mechanical energy of the system is transformed into the internal potential energy of the nanoparticle. Once the nanoparticle arrives at point $M$ (that is the beginning of the dissipation cycle, $t$ $=0)$, it jumps to the adjacent $H$ site. The latter stage is known as the slip stage. Note that this unstable equilibrium after the $M$ point occurs when the cantilever of FFM or the weakly bound nanoparticle itself have sufficiently lowelastic constants. As a matter of fact, the cantilevers of FFM

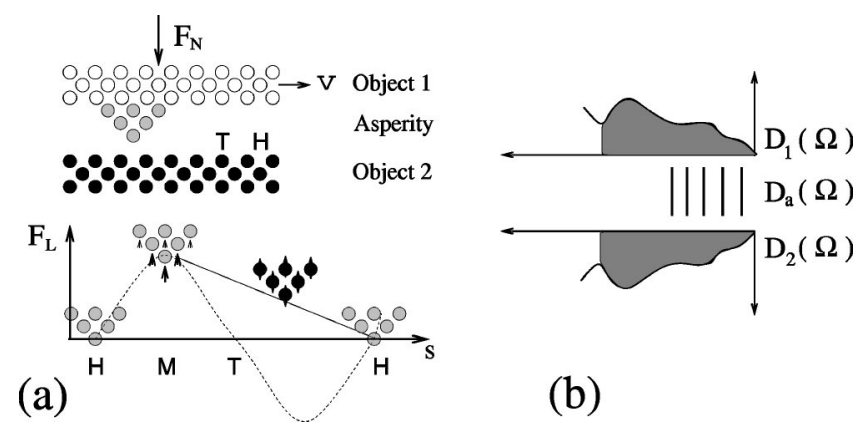

FIG. 1. (a) A schematic description of the friction system. Object 1 (empty circles), asperity or nanoparticle (shaded circles) and object 2 (black circles). $H$ and $T$ are the low- and high-energy sites, respectively, in the course the sliding of the asperity +object 1 over object 2. The deformation of the asperity in the stick step from $H$ to $M$, and vibration of its atoms in the slip step from $M$ to $T$ are shown on a force diagram. (b) Density of states of normal modes, $D_{1}, D_{a}$, and $D_{2}$, for object 1 , asperity and object 2 , respectively. 
are chosen to be especially soft in order to lead to the slip stage. Many molecules, such as $C_{60}$, or polymers, can have low stiffness constants for certain types of deformation (or modes). A sharp asperity of the object 1 itself can have lowelastic stiffness owing to the low-atomic coordination numbers of atoms at the apex. In the course of slip, external forces acting on the nanoparticle and sample vanish temporarily, so that the displaced atoms are released suddenly, 7,8 and that the atoms of the nanoparticle start to vibrate around their equilibrium positions. At the end, owing to the nonconservative nature of motion, the energy initially subtracted from the mechanical energy of the motion $\delta V_{T}$ is now expended irreversibly to excite phonons above the occupation number that had been in equilibrium at the ambient temperature $T_{o}$. Denoting the occupation numbers corresponding to $T$ and $T_{o}$ as $n_{q}$ and $n_{q}^{o}$, respectively, the excess phonon distributions are expressed as,

$$
\Delta n_{q}=n\left(\Omega_{q}, T\right)-n^{o}\left(\Omega_{q}, T_{o}\right) .
$$

Here, $q$ denotes the mode, including the polarization, and $T$ $>T_{o}$. In the present work, for the sake of simplicity, we consider energy dissipation from only a single nanoparticle. The extension of the model to account for the generation of excess phonon distributions in randomly distributed nanoparticles and at the surfaces of the macroscopic objects at the close proximity of the nanoparticle is also possible.

The nanoparticle has a discrete frequency distribution due to finite level spacings, $D_{a}(\Omega)=\Sigma_{q} \delta\left(\Omega-\Omega_{q}\right)$; the densities of states of the metal objects, $D_{1}(\Omega)$ and $D_{2}(\Omega)$, are quasicontinuous as shown in Fig. 1(b). The decay of $\Sigma_{q} \Delta n_{q}$ may occur in various ways: First, the electron-hole pairs are excited as a result of electron-phonon coupling with the rate,

$$
\begin{aligned}
& (2 \pi) / \hbar \mid\left\langle t_{k+q}+1, t_{k}-1 ; n_{q}-1\right| \\
& \quad \times\left.\Sigma_{k q} M_{k q} c_{k+q}^{+} c_{k} a_{q}\left|t_{k+q}, t_{k} ; n_{q}\right\rangle\right|^{2},
\end{aligned}
$$

in terms of fermion and boson operators. This way electrons are excited by annihilating phonons and become locally hot, and dissipate their energy through metal objects. Second, $\Sigma_{q} \Delta n_{q}$ is transferred from the nanoparticle to the adjacent objects (or samples). Here we will turn our attention to the latter, i.e., phononic dissipation and express the total vibrational Hamiltonian of the system as $H=H_{o}+H_{i n t}$, where the uncoupled part is

$$
H_{o}=\sum_{q_{1}} \hbar \Omega_{q_{1}} a_{q_{1}}^{+} a_{q_{1}}+\sum_{q_{2}} \hbar \Omega_{q_{2}} a_{q_{2}}^{+} a_{q_{2}}+\sum_{q} \hbar \Omega_{q} a_{q}^{+} a_{q} .
$$

In this equation $q_{1}, q_{2}$, and $q$ are the modes of vibration of uncoupled object 1 , object 2 , and the nanoparticle, respectively. We can express the coupling between them,

$$
\begin{aligned}
H_{\text {int }}= & \sum_{q_{1}, q} \mathcal{T}_{q_{1} q}\left(a_{q_{1}}^{+} a_{q}+a_{q_{1}} a_{q}^{+}\right)+\sum_{q_{2}, q} \mathcal{T}_{q_{2} q}\left(a_{q_{2}}^{+} a_{q}+a_{q_{2}} a_{q}^{+}\right) \\
& +\sum_{q, q^{\prime}, q_{1}} \mathcal{U}_{q q^{\prime} q_{1}}\left(a_{q} a_{q^{\prime}} a_{q_{1}}^{+}+\ldots\right)+\ldots
\end{aligned}
$$

in terms of the harmonic, $\mathcal{T}$, and anharmonic, $\mathcal{U}$, coupling constants. In the present study, we consider explicitly only the linear coupling between the nanoparticle and object 1, and neglect the smaller second and third terms in Eq. (3). We also assume that coupling between the nanoparticle and object 1 is weak. ${ }^{14}$ The saturation of the electronic states of the nanoparticle can yield also relatively weak physisorption bonds. The anharmonic coupling between the modes of the nanoparticle can be important if they are large and the level spacings of the modes are sufficiently small. Such a situation leads to a transition to ergodic behavior within the nanoparticle. $^{13,15}$ The discrete states of the nanoparticle in contact with the continuum of states of object 1 are broadened and form a Lorentzian-like local density of states; the width of each Lorentzian can be taken as the decay rate of the corresponding excitation. Certainly within object 1 there are phonon-phonon interactions, which insure that energy dissipated from the nanoparticle into the substrate is not coherently returned to the nanoparticle. The decay rate from a mode $q$ to the metal object is then

$$
\Gamma_{q}=\frac{2 \pi}{\hbar}\left|\mathcal{V}_{1 q}\right|^{2} D_{1}\left(\Omega_{q}\right)
$$

where the matrix elements $\mathcal{V}_{1 q}$ coupling states of the nanoparticle to those of the substrate are determined by the operator $H_{i n t}$, which, within the approximations described above, is specified by the first sum on the right-hand side of Eq. (3). The energy introduced into the nanoparticle by elastic deformation is expressed as,

$$
\delta V_{T}=\sum_{q} \delta V_{q}=\sum_{q} \frac{1}{2} M \Omega_{q}^{2} b_{q}^{2},
$$

where $b_{q}$ is the distance over which mode $q$ of the nanoparticle is deformed. This energy is converted into phononic energy, so that

$$
\delta V_{q}=\hbar \Omega_{q}\left(\Delta n_{q}+\frac{1}{2}\right),
$$

where $\Delta n_{q}$ is the difference between the number of phonons in mode $q$ immediately after the stick step $n_{q}$ and the number at equilibrium $n_{q}^{0}$. From the Planck distribution we can estimate an effective temperature $T$ for mode $q$ given $n_{q}$.

The initial state of the nanoparticle can be expressed now in the occupation number representation $\Psi_{i}\left(n_{1}, n_{2}, \ldots n_{q} \ldots n_{3 N}\right)$. Normally, in equilibrium at $T_{o}$ the number of quanta, which can be obtained from Planck's distribution, is $n_{q}^{o}=n\left(\Omega_{q}, T_{o}\right)$. If $\Delta n_{q}>0, \quad n_{q}\left(\Omega_{q}, T\right)$ will decay with the rate $\Gamma_{q}$ as

$$
\frac{d \Delta n_{q}}{d t}=-\Gamma_{q} .
$$

As a result, $\Psi_{i}$ being a point in the vibrational quantum number space of $3 \mathrm{~N}$ dimensions will relax to the final state, $\Psi_{f}$. Phonon-phonon interactions in the nanoparticle are allowed if anharmonic couplings are included. ${ }^{13,15}$ The anharmonic coupling constants are small, but they may change the occupation number by phonon creation (annihilation). If the overall coupling is sufficiently weak so that $\Gamma_{q}$ is sufficiently small, the temperature of the nanoparticle or lubricant molecule increases as it continues to slide. The coupling constants in Eq. (4) can be determined, for example by carrying out $a b$ initio frozen phonon calculations. We estimate them 
simply by using the scaling arguments of Gruebele and coworkers, ${ }^{16}$ i.e., $\mathcal{T}_{q q_{1}}=\eta\left(\epsilon_{q} \epsilon_{q_{1}}\right)^{1 / 2}$, where $\epsilon_{q}=\hbar \Omega_{q}$ and $\epsilon_{q_{1}}=\hbar \Omega_{q_{1}}$. The dimensionless coefficient $\eta$ is system specific; it is often $\eta \sim 10^{-2}-10^{-3}$ in molecules, but of course would vary with the nature of the bonding. We used $\eta$ $=0.0023$ in the calculations below. Then $\left|\mathcal{V}_{1 q}\right|^{2}$ in Eq. (4) is $\eta^{2} \epsilon_{q}^{2} n_{q}\left(n_{q}+1\right)$, where we take the energy and occupation numbers of nanoparticle mode $q$ and object 1 mode $q_{1}$ to be equal. We calculate the density of states of object $1, D_{1}(\Omega)$, using the Debye approximation, $D_{1}(\Omega)=\sum_{j} V \Omega^{2} / 2 \pi^{2} v_{j}^{3}$, where $V$ and $v_{j}$ are the volume of object 1 , and velocity of sound for a given polarization $j$, respectively. Finally, by defining,

$$
R\left(\Omega_{q}\right)=\frac{V}{\hbar v_{j}^{3}} \eta^{2} \epsilon_{q}^{4}\left(n_{q_{1}=q}+1\right)
$$

we reexpress the rate of decay as, $\Gamma_{q}=R\left(\Omega_{q}\right) n_{q}$. Accordingly, $d n_{q}(t) / d t=-R\left(\Omega_{q}\right) n_{q}(t)$. This leads to the solution,

$$
n_{q}(t)=n_{q}^{o}+n_{q}(t=0) \exp \left[-R\left(\Omega_{q}\right) t\right],
$$

where $n_{q}(t=0)$ is the number of phonons at the beginning of the slip stage. Similarly, the decay of $\delta V_{T}$ is found to be

$$
\delta V_{T}(t)=\sum_{q} \delta V_{q}(t=0) \exp \left[-R\left(\Omega_{q}\right) t\right]
$$

Here, again, $\delta V_{T}(t=0)$ is the elastic deformation energy stored in the asperity or lubricant molecule at the end of the stick stage. From Eqs. (8)-(10) we see that, due to the low density of states of the substrate at low frequencies, dissipation from low-frequency modes of the nanoparticle is relatively slow.

\section{NUMERICAL CALCULATIONS AND DISCUSSION}

We next present a quantitative analysis of energy dissipation by applying the above theory to a simple system consisting of a sharp asperity that includes $N=14 \mathrm{Cu}$ atoms (having $1+4+9$ atoms in the consecutive planes) and is attached to a metal. In order to compute the vibrational normal modes, we first obtain the interatomic force constants from a realistic empirical potential, ${ }^{17}$ and then we form the dynamical matrix of the asperity. The eigenvectors and eigenfrequencies are calculated by diagonalizing the dynamical matrix. The elastic deformation of the asperity under the constant loading force, and hence, the lateral and perpendicular displacements of its atoms at point $M$ (just before the slip stage) can be calculated by using $a b$ initio methods, or classical molecular statics. ${ }^{7}$ Here, for the sake of demonstration, we assume that the first- (apex) and second-layer atoms of the asperity (which have rather low-coordination number) are displaced by 0.3 and $0.15 \AA$, respectively. With these displacements of the atoms of the asperity attached to object 1 , we determine $b_{q}$ and $n_{q}$ for the initial state defined above, $\Psi_{i}\left(\left\{\mathbf{u}_{\mathbf{j}}\right\}\right)$; then following the steps described through Eq. (10), we calculate the energy dissipation in friction.

Figure 2 illustrates the decay of four consecutive lowfrequency modes and the decay of $\delta V_{T}(t)$, defined by Eq. (10). The mode having the lowest frequency, $\Omega_{q=1}$, with
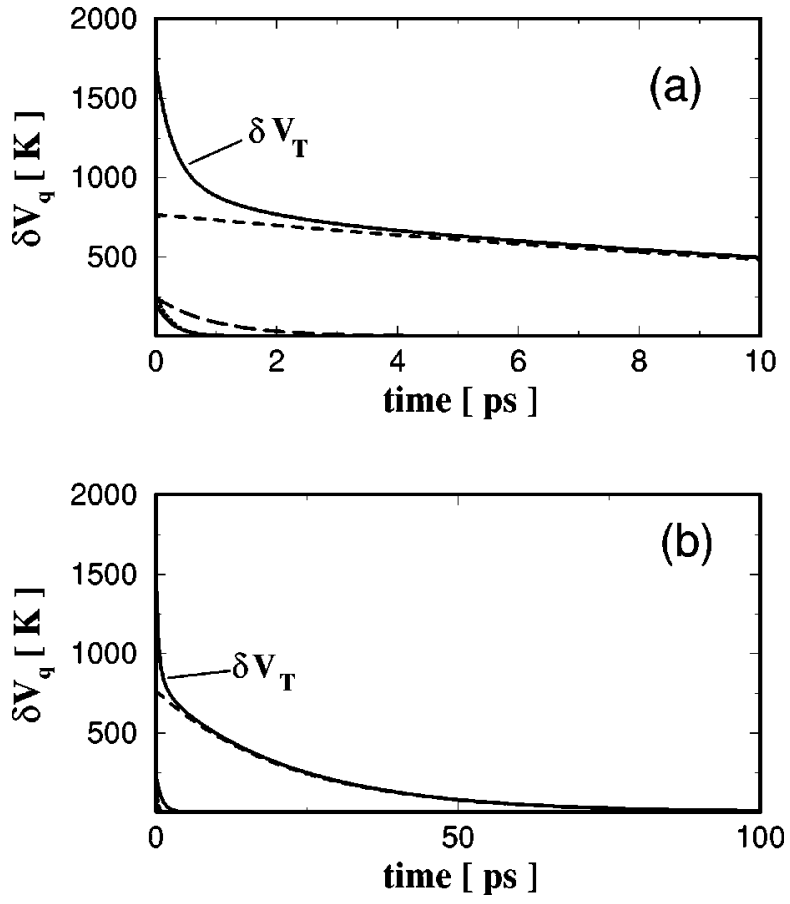

FIG. 2. The phononic energy dissipation from an asperity or a nanoparticle for the $\mathrm{Cu}$ system described in the text. Plotted is the decay of vibrational energy from the four lowest frequency modes. (a) Time scale up to $10 \mathrm{ps}$, (b) time scale up to $100 \mathrm{ps}$.

energy $\epsilon_{q=1}=\hbar \Omega_{q=1}=89.7 \mathrm{~K}$, has the highest occupation number, $n_{q=1} \approx 9$. The temperature of this excitation estimated using the Planck distribution corresponds to $T$ $=809 \mathrm{~K}$. Due to the fourth power of $\epsilon_{q}$ in Eq. (8) this mode has also the smallest decay rate, $\Gamma_{q=1}=0.41 \times 10^{11} \mathrm{~s}^{-1}$ and therefore dominates the decay rate of $\Sigma_{q} \Delta n_{q}$ and $\delta V_{T}$. Higher frequency modes decay faster, and thus influence the overall decay rate much less, as seen in Fig. 2.

For the data plotted in Fig. 2, we observe that $\delta V_{T}$ almost vanishes within $100 \mathrm{ps}$ (or $10^{-10} \mathrm{~s}$ ). From the usual effective lateral force constant and the corresponding effective frequency $\nu_{e}$, the slip time $\tau$, from $M$ to $H$ in Fig. 1(a) is estimated to be much longer than $1 \mathrm{ps}$. For example, $\nu_{e}$ $\sim 200 \mathrm{~K} \mathrm{~Hz}$ for the soft cantilever used in friction force microscopy. This justifies the assumption made in the early treatment of friction by Tomanek and coworkers ${ }^{8}$ that the phononic excitation generated after stick is totally dissipated in the following slip step. Still, for the much faster fly times of weakly bound asperities or lubricant molecules, we might find competition with the rate of phononic dissipation. Moreover, the dimensionless coupling parameter, $\eta$, may well be much smaller than the value $\eta \approx 0.002$ used in our calculations, which, as seen from Eq. (8), would slow dissipation. We note also that if the harmonic coupling were strong, the modes of the asperity and the object would form current transporting states $Q$. In the quantum heat transfer picture, ${ }^{18-20}$ it has been shown that the (phononic) thermal conductance of such a state is limited by $\mathcal{K} \sim k_{B} v_{j} x^{2} e^{x}\left(e^{x}\right.$ $-1)^{-2}$ with $x=\hbar \Omega_{Q} / k_{B} T$. This implies that even in the strong coupling case the temperature of the asperity may increase with friction if the elastic deformation is large, due to the finite rate of phonon dissipation. 
Other quantitative studies related with energy dissipation in friction have addressed the role of phononic dissipation through lubricant films, mainly a regular layer of noble gas atoms adsorbed on a noble metal surface. The energy dissipation, friction force and $\tau$, were calculated. The work described in Ref. 12 studied the molecular origin of friction in an ideal system, a layer of adsorbed $\mathrm{Kr}$ atoms sliding over the $\mathrm{Ag}, \mathrm{Au}$ (111) surface by performing molecular dynamics and perturbation calculations. The success of the calculations in reproducing the measured ${ }^{21}$ variation of $\tau$ are taken as evidence that anharmonic coupling and hence phononic dissipation is the major source of friction. Reference 13(a) describes a perturbation theory treatment and calculation of energy dissipation due to phonon excitations that contribute to friction between a film and substrate. Reference 13(b) is concerned with energy dissipation in a small size solid interacting with harmonic and anharmonic forces and forced to vibrate harmonically from one end, which could model forcing due to sliding over another substrate. The mesoscopic solid is represented by a linear chain. Owing to the large mode frequency spacings the time average of the calculated energy of the chain was unchanged indicating lack of energy dissipation. However, as the size of the atomic chain increases beyond a critical value there occurs a transition from a nondissipative to a dissipative regime. These results are qualitatively consistent with other studies describing quantum mechanically the localization-delocalization transition in finite, many-dimensional coupled nonlinear oscillator systems. ${ }^{15}$ The possibility of nonergodicity of the mesoscopic solid was interpreted as evidence of nearly frictionless sliding for a sufficiently small solid. More recently, Tomassone et al. ${ }^{22}$ performed molecular-dynamic simulations for a Xe film sliding on an $\operatorname{Ag}(111)$ surface and concluded that the phonon excitations in the film dominates the friction, consistent with the results of earlier theoretical works. ${ }^{12,13}$ Caroli and Nozieres ${ }^{23}$ proposed a macroscopic mechanism of dry friction that results from the elastic response of the asperities in the presence of the bistability. By using continuum elasticity theory they obtained the hysteresis cycles qualitatively. The atomic scale study of the same issue including the plastic deformation or wear have been performed extensively by using simulations based on the classical molecular dynamics together with realistic potential functions (see for example Refs. 1,4, and 7).

Our paper presents a microscopic theory that explains how energy dissipation and hence friction occurs in a system having either a single asperity or single lubricant molecule that are weakly bound to a substrate. Therefore, it is different from the above studies. ${ }^{12,13,22}$ The present physical model fits very well to friction force microscopy, ${ }^{2}$ and the present analysis can be extended to multiple asperities or to boundary lubrication with lubricant molecules random in size and orientation. The theory provides a clear picture of phononic energy dissipation through a nanoparticle, and allows quantitative analysis on the atomic scale. Here, emphasis is placed on energy transfer through the interface between the nanoparticle and the substrate, assuming coupling between them is not too strong. As for strong coupling, we expect that ballistic heat transfer ${ }^{18-20}$ with quantum features would play a crucial role in the dissipation process.
*Permanent address: UCSD Department of Chemistry and Biochemistry, La Jolla, CA 92093-0339.

${ }^{1}$ For further references see Physics of Sliding Friction, Vol. 311 of NATO Advanced Studies Institute, Series E: Applied Sciences, edited by B. N. J. Persson and E. Tosatti (Kluwer, Dordrecht, 1996); Micro/Nanotribology and its Applications, Vol. 330 of NATO Advanced Studies Institute, Series E: Applied Sciences, edited by B. Bhushan (Kluwer, Dordrecht, 1997).

${ }^{2}$ C. M. Mate, G. M. McClelland, R. Erlandsson, and S. Chang, Phys. Rev. Lett. 59, 1942 (1987).

${ }^{3}$ G. A. Tomlinson, Philos. Mag. 7, 905 (1929); J. Frenkel and T. Kontorova, Phys. Z. Sowjetunion 13, 1 (1938).

${ }^{4}$ B. Bhushan, J. N. Israelachvili, and U. Landman, Nature (London) 347, 607 (1995).

${ }^{5}$ A. P. Sutton and J. B. Pethica, J. Phys.: Condens. Matter 2, 5317 (1990); J. A. Nieminen, A. P. Sutton, and J. B. Pethica, Acta Metall. Mater. 40, 2503 (1992).

${ }^{6}$ M. R. Sorensen, K. W. Jacobsen, and P. Stoltze, Phys. Rev. B 53, 2101 (1996); M. R. Sorensen, K. W. Jacobsen, and H. Jonsson, Phys. Rev. Lett. 77, 5067 (1996).

${ }^{7}$ A. Buldum and S. Ciraci, Phys. Rev. B 55, 2606 (1997); 55, 12 892 (1997); A. Buldum, S. Ciraci, and I. P. Batra, ibid. 57, 2468 (1998).

${ }^{8}$ W. Zhong and D. Tomanek, Phys. Rev. Lett. 64, 3054 (1990); D. Tomanek, W. Zhong, and H. Thomas, Europhys. Lett. 15, 887 (1991).

${ }^{9}$ L. S. Levitov, Europhys. Lett. 8, 449 (1989).
${ }^{10}$ B. N. J. Persson, Phys. Rev. B 44, 3277 (1991); B. N. J. Persson and A. I. Volotkin, J. Chem. Phys. 103, 8679 (1995).

${ }^{11}$ A. Dayo, W. Alnasrallah, and J. Krim, Phys. Rev. Lett. 80, 1690 (1998).

${ }^{12}$ M. Cieplak, E. D. Smith, and M. O. Robins, Science 265, 1209 (1994); E. D. Smith, M. O. Robbins, and M. Cieplak, Phys. Rev. B 54, 8252 (1996).

${ }^{13}$ (a) J. B. Sokoloff, Phys. Rev. B 42, 760 (1990); 42, 6745(E) (1990); 51, 15573 (1995); (b) J. B. Sokoloff, Phys. Rev. Lett. 71, 3450 (1993).

${ }^{14}$ Owing to the relatively low-coordination number of atoms at the contact the coupling between the nanoparticle and the object 1 can be weak. An oxide layer can also increase the spacing between a nanoparticle and the object 1 and hence, weaken the coupling between them. Accordingly, the vibration states of nanoparticle and the object 1 are calculated separately as expressed in Eq. (2) and the energy transfer (dissipation) from the nanoparticle to the object 1 is treated as outlined by Eqs. (3)(4).

${ }^{15}$ D. E. Logan and P. G. Wolynes, J. Chem. Phys. 93, 4994 (1990); D. M. Leitner and P. G. Wolynes, ibid. 101, 541 (1996); Chem. Phys. Lett. 280, 411 (1997).

${ }^{16}$ D. Madsen, R. Pearman, and M. Gruebele, J. Chem. Phys. 106, 5874 (1997).

${ }^{17}$ S. Erkoc, Z. Phys. D 32, 257 (1994); H. Mehrez, S. Ciraci, C. Y. Fong, and S. Erkoc, J. Phys.: Condens. Matter 9, 10843 (1997). 
${ }^{18}$ L. G. C. Rego and G. Kirczenow, Phys. Rev. Lett. 81, 232 (1998).

${ }^{19}$ D. E. Angelescu, M. C. Cross, and M. L. Roukes, Superlattices Microstruct. 23, 673 (1998).

${ }^{20}$ A. Buldum, S. Ciraci, and C. Y. Fong (unpublished).

${ }^{21}$ J. Krim, D. H. Solina, and R. Chiarello, Phys. Rev. Lett. 66, 181 (1991).
${ }^{22}$ M. S. Tomassone, J. B. Sokoloff, A. Widom, and J. Krim, Phys. Rev. Lett. 79, 4798 (1997).

${ }^{23}$ C. Caroli and P. Nozieres in Physics of Sliding Friction, edited by B. N. J. Perssons and E. Tosatti, Vol. 311 of NATO Advanced Studies Institute, Series E: Applied Sciences (Kluwer, Dordrecht, 1996), p. 27. 